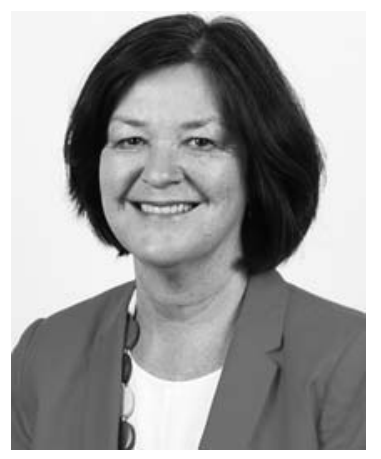

\title{
A Conversation with Susan Clark
}

\author{
INTERVIEWER: MiRNA KVAJO \\ Scientific Editor, Cell
}

Susan Clark is a Senior Principal Research Fellow and Head of the Division of Genomics and Epigenetics at the Garvan Institute of Medical Research.

\begin{abstract}
Mirna Kvajo: You've studied the role of epigenetics in normal cells' behavior and function and also in disease; it's fair to say that you are one of the pioneers in epigenetics. You're one of the first people who studied changes such as DNA methylation, and you also were the first to develop a sequencing method that allows one to look at methylation patterns. Can you tell me how you came into this field just as it was developing?
\end{abstract}

Dr. Clark: My research science journey began in the mid 1970s, when I started my undergraduate degree in Molecular Biology at the Australian National University, with the lofty goal to understand how genes are regulated. Restriction enzymes, ligation, and cloning had just been discovered. My Ph.D. in the late '70s at the University of Adelaide was to clone human histone genes for the first time and sequence them. We were using Maxam-Gilbert sequencing in those days, and you could read the bases on acrylamide gels. I noticed that the migration of the bands was different at CCG sites, and we wondered whether that difference in migration meant that the cytosines in this context were methylated.

In the 1980s restriction enzymes were found to be useful to detect DNA methylation as well, but clearly could only be used to detect methylation at a limited number of $\mathrm{CpG}$ sites. I joined the laboratory of Marianne Frommer at CSIRO Division of Molecular Biology, and she was also interested in DNA methylation. It took until the early ' 90 s to solve the problem of how to assess methylation of individual $\mathrm{CpG}$ sites in a different way. The eureka moment for Marianne came from reading two new papers in the literature, one describing the chemistry of sodium bisulfite conversion of cytosine residues to uracil residues and the second the development of PCR (polymerase chain reaction). By combining these two technologies with sequencing, we showed that we could detect DNA methylation at single-molecule resolution. Simply sequencing amplified DNA after bisulfite conversion allowed you to read whether DNA was methylated or not and most importantly which CpG sites were modified in any different loci and cell type.
Bisulfite PCR sequencing totally changed what we thought about the role of DNA methylation. In 1975, Art Riggs and Robert Holliday proposed methylation might have a role in gene regulation. That hypothesis is what really got me into the field in the first place, but because the technology was limited to restriction enzymes, it was very difficult to address. Also, the field really didn't get much traction because in the ' 80 s it was shown that the main models used for molecular biology research, Drosophila and worms, don't have DNA methylation, therefore how could it be important in the control of gene regulation? Suddenly, the hypothesis went out of favor.

It was therefore really fantastic to develop a new method that could be used to look at the methylation pattern of every cytosine residue in any sequence. That was the first time we and others could map methylation patterns in different cell types and demonstrate their importance in gene expression. Critically at the same time, the DNA methyltransferase enzymes were knocked out in mouse and these knockouts were shown to be lethal. The combination of showing methylation was essential for development, and a new method to detect individual cytosine sites really opened up interest in the field again.

Mirna Kvajo: You started by looking at this in a very fundamental way to understand this process of modifications through DNA, but at the same time there was the awareness that this could be important for pathological states. You're working on cancer now.

Dr. Clark: I am now, but that took a long time. Initially, we started looking at methylation profiles at $\mathrm{CpG}$ islands and showed that they are typically unmethylated regardless of the cell types, whereas tissue-specific promoters are differentially methylated in different cell types. Then we looked during embryo development to see how the methylation of candidate genes changes from fertilization to implantation. We did that work in the late 1990s. It was very difficult then to get funding to do basic research, as it

(C) 2016 Clark. This article is distributed under the terms of the Creative Commons Attribution-NonCommercial License, which permits reuse and redistribution, except for commercial purposes, provided that the original author and source are credited. 
still is now. That's when we decided to see if methylation changes during the course of a disease.

That was a very good transition to make. I was inspired with the first report that the retinoblastoma protein is mutated in retinoblastoma tumors. I asked the question: Could the promoter $\mathrm{CpG}$ island also be methylated? One of the key investigators in the field sent us DNA from retinoblastoma tumors. We did bisulfite sequencing on the retinoblastoma promoter $\mathrm{CpG}$ island and showed that many of the retinoblastoma tumors had one allele methylated and one that was mutated. It demonstrated for the first time that it wasn't just genetic mistakes in these key driver tumor-suppressor genes; methylation was also a bona fide way of switching off a tumor-suppressor gene.

Mirna Kvajo: That complicated things for people who were hoping to look at cancer as a purely genetic disease.

Dr. Clark: There's still an active debate between genetic and epigenetic researchers about this! However from that first example, the field opened up. We and others showed that it's not just single genes that become methylated; many hundreds, even thousands, of genes get differentially methylated and repressed. The next question we asked, "Could there be regions of contiguous gene inactivation in cancer as a result of methylation?" It was very surprising at the time to show that not just single $\mathrm{CpG}$ island promoters were methylated, but megabase regions harboring neigboring $\mathrm{CpG}$ islands were hypermethylated in cancer. This result showed that there must be a program eliciting regional changes in epigenetic reprogramming across the cancer genome.

Mirna Kvajo: Did you look at other cancer types? Is there a general idea about how much methylation changes in cancer or in different cancer types?

Dr. Clark: Yes. Initally we were fairly opportunistic in selecting cancer types where we could collaborate with enthusiastic surgeons and oncologists. We moved from retinoblastoma tumors to leukemia and then to prostate and breast cancer. Now with the success of the ICGC (International Cancer Genome Consortium) and TCGA (The Cancer Genome Atlas) programs, it is well established that DNA methylation changes are widespread in cancer, even if each clinical sample and subtype of cancer might have a different level and profile of DNA methylation aberrations.

Mirna Kvajo: Do you work on other DNA modifications that have been implicated in the context of cancer?

Dr. Clark: One of the key questions that is still not solved in the cancer field is what elicits the opposing changes in DNA methylation? In cancer, you have global hypomethylation - so, loss of methylation - in addition to the gain: hypermethylation in $\mathrm{CpG}$ islands. We're interested in understanding the cause of that and the interrelation with histone marks and replication timing. It is now possible to be able to overlay the DNA methylation landscape with overarching histone modifications to see what is the correlation between methylation and chromatin remodeling, both in normal cells and in the cancerous state.

Mirna Kvajo: The field of epigenetics in general has a big influx of new techniques (and new adjustments and improvements of techniques) that allows this global analysis all the time. We could start a project at one point and, in the meantime, there are several iterations of how you could do it differently.

Dr. Clark: Well, recombinant DNA technology was driven by restriction enzymes and cloning. I think all fields are driven by innovations in technology, but this field of epigenomics in particular is really moving at such a fast pace. You think you've got a great result with a new technique, and suddenly it can be surpassed and the reviewers or referees will say "Why don't you try this as well?"

I think a game changer is a technology that allows you to manipulate or perturb the cell to induce malignancy in vivo in a controlled setting. The problem with looking at cancer is that by the time we can identify the cancer-by the time it's large enough for the surgeon to take it outall of these epigenetic changes have already happened. It's very difficult. We really need a way to either follow the cell's journey from the premalignant state to the cancer state or a way of manipulating the normal cells with what we think are drivers of epigenetic change in cancer so we can catalog the temporal progression and understand what comes first - genetic or epigenetic lesions.

Personally, it would be preferable to avoid mouse models. Human cancers are really quite unique because it takes a long time for a cancer to grow, especially prostate cancer. Ideally, we need model systems in a human that we can follow, and that's where single-cell technology is a game changer. Methylation is heterogeneous in disease, and to be able to do single-cell technology and follow those cells as they evolve and migrate is really fantastic. CRISPR (clustered regularly interspaced short palindromic repeats) is another game-changing technique that allows you to manipulate epigenetic marks one at a time. Rather than just profiling and looking at patterns in our normal and cancer cell sequencing data, we now have the opportunity to be able to manipulate and hopefully to see what elicits these epigenetic patterns in the first place.

Mirna Kvajo: You've used another technique that moves a step beyond looking at DNA to the whole genome. Changes in chromatin conformation in cancer confer some properties that are different from the normal properties of the cell. Why should they care how the chromatin in the genome is folded, and why are cancer cells different?

Dr. Clark: We commonly just think of the genome as linear, because that's how the sequence is when we visualize it on a page. We have to remember that DNA isn't linear. It is folded in this incredibly complex and exquisite way inside the nucleus, and one of the challenges has been to determine what that structure is inside the nucleus. The development of Hi-C chromatin conforma- 
tion sequencing technology has allowed us for the first time to get a picture, a snapshot, of how different regions of the chromatin interact with each other inside the nucleus. We were very interested to see if this conformation changes in cancer. We used Hi-C technology and compared normal cells and cancerous prostate cells to look at the topological domains and where the changes might be.

Mirna Kvajo: When it comes to their genome, cancer cells change on so many levels, and the way some transcriptional programs are going to happen or not really seems to depend on all of these factors.

Dr. Clark: It's all interdependent. Simply, the genome is quite de-arranged in cancer, so it's not surprising that has an impact on the conformation. One of the biggest surprises was that the basic structure of the core topological domains is intact. I would have predicted we would see a lot more disruption, but maintaining this core structure appears essential for cell viability. Even though the cancer has gone awry in many ways, I don't think the 3D genome can afford to totally disrupt its fundamental "origami" shape that defines a cell.

The other part of our finding is that where we do identify structural changes in cancer, these involve promoters and enhancers forming new contacts not found in normal cells. The new contacts facilitate ectopic expression of cancer genes. These new insights into alterations of the three-dimensional architecture in cancer have only been possible since the development of new ways of visualizing chromatin interactions. 


\section{$\$_{\text {CSH\& }}^{\infty}$ Cold Spring Harbor Symposia SYMPOSIA On Quantitative Biology}

\section{A Conversation with Susan Clark}

Cold Spring Harb Symp Quant Biol 2016 81: 306-308 originally published online March 13, 2017 Access the most recent version at doi:10.1101/sqb.2016.81.031948

Creative This article is distributed under the terms of the

Commons http://creativecommons.org/licenses/by-nc/4.0/, which permits reuse and

License redistribution, except for commercial purposes, provided that the original author and source are credited.

Email Alerting Receive free email alerts when new articles cite this article - sign up in Service the box at the top right corner of the article or click here. 